Review Article

\title{
The Role of Fatigue in the Aging Swallow: A Review
}

Danielle Brates ${ }^{1,{ }^{+},{ }^{*}}$, Michelle S. Troche ${ }^{2,+}$, Sonja M. Molfenter ${ }^{1,+}$

1. Department of Communicative Sciences and Disorders, New York University, 665 Broadway, New York, NY 10012, USA; E-Mails: db2814@nyu.edu; smm16@nyu.edu

2. Laboratory for the Study of Upper Airway Dysfunction, Department of Biobehavioral Sciences, Teachers College, Columbia University, New York, NY, 10027, USA; E-Mail: mst2139@tc.columbia.edu

+ These authors contributed equally to this work.

* Correspondence: Danielle Brates; E-Mail: $\underline{\mathrm{db} 2814 @ \text { nyu.edu }}$

Academic Editor: David G Smithard

Collection: Dysphagia in the Elderly

\section{OBM Geriatrics}

2021, volume 5 , issue 2

doi:10.21926/obm.geriatr.2102166
Received: October 29, 2020

Accepted: April 02, 2021

Published: April 15, 2021

\begin{abstract}
Fatigue is a term commonly used to describe patient performance and/or subjective experience in the evaluation and management of swallowing disorders (known as dysphagia). There is an association between fatigue and aging, as well as fatigue and many dysphagiacausing diseases/disorders. Therefore, speech-language pathologists (SLPs) are justifiably aware of and concerned about the potential impact of fatigue on swallowing performance and mealtime behavior. However, there is minimal agreement on and understanding of what constitutes swallowing-related fatigue, how it is identified and measured, who is at risk, and its impact on swallowing function, overall health, and quality of life. The purpose of this review is to discuss the role of fatigue in swallowing and eating behavior in the context of aging, and how fatigue may be measured and managed clinically. We review the concept of fatigue and its clinical implications for swallowing function and mealtime behavior through the dichotomous framework of self-perceived fatigue versus measurable fatigability. Quantitative
\end{abstract}

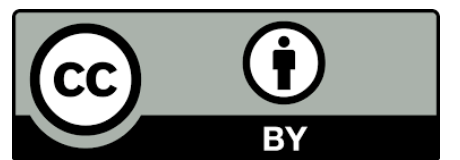

(c) 2021 by the author. This is an open access article distributed under the conditions of the Creative Commons by Attribution License, which permits unrestricted use, distribution, and reproduction in any medium or format, provided the original work is correctly cited. 
fatigability and patient-reported fatigue are discussed. We conclude with implications for future research.

\section{Keywords}

Dysphagia; aging; swallowing-related fatigue; fatigability

\section{Introduction}

\subsection{Fatigue in Aging}

Fatigue is frequently reported by older adults and is a common reason for seeking medical care $[1,2]$. Fatigue in older adults can be extremely debilitating as it may interfere with activities of daily living (e.g. eating, dressing, hygiene [3] and quality of life [4, 5], and is associated with higher risk for disability onset [6]. Associations between fatigue and many medical conditions have been well established, including cancer [7], stroke [8], multiple sclerosis [9], chronic obstructive pulmonary disease [10], and osteoarthritis [11], yet fatigue may also present in the absence of any definitive cause [12]. The prevalence of fatigue is difficult to estimate, but has been reported to affect between 20 and 55\% of community-dwelling older adults [13], and as many as $98 \%$ of individuals living in residential care facilities [14]. Together with unintentional weight loss, weakness, slow walking speed, and low physical activity, fatigue is a key clinical indicator of frailty [15]. Importantly, a diagnosis of frailty is most prevalent among older adults [16], and is associated with increased risk for adverse health outcomes, mortality [15] and dysphagia, (i.e. swallowing impairment) [17, 18], which is the focus of this review.

\subsection{Introduction to Swallowing-Related Fatigue}

Consuming a meal is generally considered an enjoyable, even relaxing, and often social experience for healthy, non-dysphagic individuals. Yet, meal consumption involves a host of complex motor, cognitive, homeostatic, psychosocial, and cultural factors that must interact to result in a safe, efficient, and pleasurable experience [19-22]. Impairments in any part of this process can lead to reduced mealtime safety [23], health (e.g. malnutrition and/or dehydration) [24, 25] and quality of life [26, 27]. Impairments may also impact an individual's eating behavior, which collectively refers to food choices and motives, feeding practices, dieting, and eating related problems such as eating and feeding disorders [28]. Swallowing is central to the act of meal consumption, and encompasses the entire process from oral preparation of the food or liquid (e.g. chewing), to transporting it from the oral cavity, through the pharynx, past a closed larynx, and into the esophagus [29]. Continual swallowing over the course of a meal can be considered an endurance task, given that, in addition to sustained attention, it requires sustained, repetitive and continuous submaximal performance (i.e. requiring only a fraction of total force capacity) of a wide array of muscles [30]. The muscles involved in safe and efficient swallowing include lingual, facial, palatal, pharyngeal, laryngeal, and respiratory muscles (see Kent [31] for a detailed review of craniofacial and laryngeal muscle structure and function). These muscles include both Type I, slow twitch, 
fatigue resistant and Type II, fast twitch, muscle fiber types, given their complex and important role for breathing, swallowing, and communicating [32-35].

Because swallowing and eating require endurance, impaired endurance, or easy fatigability, has the potential to negatively affect this vital human behavior. An individual's ability to safely and efficiently consume a complete meal could potentially be impacted by either declines in swallowing performance increasing risk for aspiration (i.e. airway invasion) and/or the need to stop prematurely due to fatigue, thus increasing the risk for malnutrition and impaired quality of life. These considerable health risks may be amplified in older adults and dysphagic populations in whom fatigue is common, including those with frailty, peripheral neurological disease (e.g. Myasthenia Gravis), central neurological disease (e.g. stroke, Parkinson's Disease), and cognitive impairment (e.g. Alzheimer's Disease, dementia). Individuals with psychological disorders (e.g. depression) are also at risk for meal-related fatigue due to changes in motivation, mood, and appetite [36, 37].

Yet, the relevance of fatigue during swallowing may be underappreciated due to the nature of how dysphagia is evaluated and diagnosed. Clinical and instrumental evaluations assess a brief "snapshot" of swallowing performance during a few sips and bites of liquids and foods. Whether this snapshot is representative of swallowing performance over the course of a full meal is a frequent source of discussion and speculation among dysphagia researchers and speech-language pathologists (SLPS), and represents a limitation in dysphagia evaluation and diagnosis [38]. Thus, while considerable attention has been given to researching the impact of force and pressure generation of lingual [e.g. 39-41] and pharyngeal [e.g. 39, 42, 43] swallowing musculature, as well as skill-based physiological parameters [e.g. 44, 45] on dysphagia and dysphagia rehabilitation, the potential role of fatigue in dysphagia is largely unknown and understudied.

Fundamental questions that need to be answered include how to define swallowing-related fatigue, and how fatigue affects swallowing physiology and function. Further, it is important to understand whether swallowing-related fatigue can be quantified to determine thresholds for risk, and the nature of the relationship between the patient-reported fatigue and measurable fatigability during swallowing are gaps in current knowledge.

\subsection{Swallowing-Related Fatigue: Gaps in Knowledge}

To understand the current state of the field of speech-language pathology regarding clinical perspectives on swallowing-related fatigue, our lab recently conducted a survey of certified SLPS who evaluate and treat adult dysphagia [46]. We found that $86 \%(n=311)$ of SLPs consider fatigue to be an important consideration in dysphagia assessment, and $45 \%$ reported explicitly evaluating fatigue during clinical swallowing evaluations. Yet, there was wide variability in how clinicians defined swallowing-related fatigue, indicating disagreement on fatigue definitions and clinical markers. Further, the most common methods for evaluating swallowing-related fatigue were through general, unspecified declines in performance, and via patient report. This lack of specificity and variability in clinical practice reflect a similar lack of standardization for fatigue definitions and measurement methods more broadly across clinical fields [47, 48]. Fatigue is difficult to define and characterize, and its clinical presentations and patient-reported symptoms can vary widely [49].

In treating patients with dysphagia, clinicians may make recommendations such as consuming smaller, more frequent meals to avoid fatigue, and will consider fatigue during dysphagia treatment planning, with such modifications as scheduling sessions at times when patients are less likely to be 
tired, and shortening session durations to minimize fatigue effects [50, 51]. In the United States, this practice is supported by the American Speech-Language-Hearing Association (ASHA), which recommends assessing the impact of fatigue on swallowing across different assessment modalities (clinical swallowing evaluations, videofluoroscopic swallow studies (VFSS), and fiberoptic endoscopic evaluation of swallowing (FEES)), but does not specify guidelines or provide resources for how SLPs should do so [52]. Clearly, there is a need to establish a common framework for conceptualizing swallowing-related fatigue to facilitate more accurate identification and measurement of fatigue in the clinical realm, and to guide future research on swallowing-related fatigue and its implications for older and dysphagic individuals.

A framework that may be highly useful for defining and characterizing fatigue in the context of swallowing is that proposed by Kluger, Krupp, and Enoka [53], who outline various factors related and contributing to fatigue (Figure 1). This framework distinguishes between the subjective experience of fatigue, or "perceived fatigue", and measurable changes in performance, or "performance fatigability" [53]. Factors contributing to perceived fatigue include homeostatic and psychological factors, whereas performance fatigability may have peripheral or central nervous system origins. Though presented as a dichotomy, the framework's developers note that perceived fatigue and performance fatigability interact with one another (to be discussed in detail later). This framework will serve as a guide through the existing literature on swallowing-related fatigue, and facilitate the identification of gaps in current knowledge.

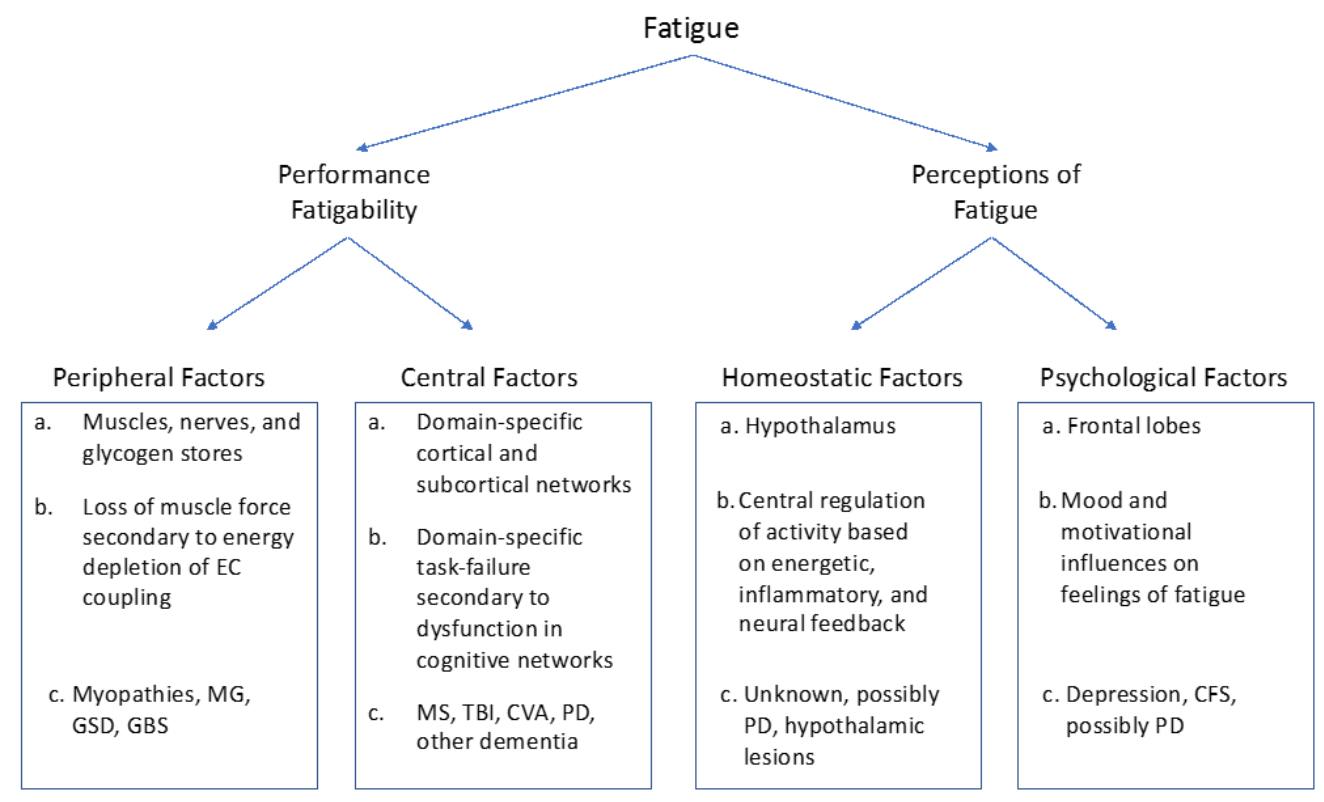

Figure 1 Figure adapted from Kluger BM, Krupp LB, Enoka RM. Fatigue and fatigability in neurologic illnesses: proposal for a unified taxonomy. Neurology. 2013; 80(4): 409-16, p. 412. Letters in boxes refer to the following: a) known neuroanatomic sites mediating this factor; b) normal function of this factor; c) pathologic states involving this factor. $\mathrm{CFS}=$ chronic fatigue syndrome; CVA = cerebrovascular accident; $\mathrm{EC}$ = excitation/ contraction; GBS = Guillain-Barre Syndrome; GSD = glycogen storage diseases; MG = myasthenia gravis; $\mathrm{MS}=$ multiple sclerosis; $\mathrm{PD}=$ Parkinson diseases; $\mathrm{TBI}=$ traumatic brain injury. 


\subsection{Current Study}

Understanding perceived fatigue and performance fatigability during swallowing will advance clinical management of patients with dysphagia by allowing SLPs to identify and properly manage fatigue-associated risks to swallowing and swallowing-related quality of life. The purpose of this review is to discuss the role of fatigue in swallowing function and eating behavior in the context of aging, propose methods for how fatigue may be measured and managed clinically, and outline implications for future research.

\section{Performance Fatigability During Swallowing: Quantitative Changes in Performance}

Performance fatigability describes the "magnitude or rate of change in a performance criterion relative to a reference value over a given time of task performance or measure of mechanical output" [53]. Both physical (i.e. motor) and cognitive fatigability can be measured, and both may be relevant to swallowing performance and rehabilitation in elderly adults.

\subsection{Effects of Motor Fatigability on Swallowing}

Declines in muscle strength with aging have been well established, both in the limbs e.g., [54-56], and in the swallowing musculature e.g., $[57,58]$. This may be related to greater age-related atrophy of fast-twitch (type II) fibers compared to slow twitch (type I) fibers in humans [59]. However, less is known about changes in muscle fatigability with aging.

In limb muscle literature, some studies have shown that fatigue resistance is higher in older adults compared to younger adults [60-62], while others demonstrate similar fatigability across ages [63-65], or less resistance to fatigue with aging [66-69]. Conflicting findings may be related to differences in the muscles studied, type of exercise performed (e.g. isometric versus isokinetic), and varied methods of measuring fatigue [70]. Evidence from the limb muscles can be helpful for guiding and informing research on swallowing-related fatigue because limb muscle fatigue has been more heavily studied e.g., [71-73]. However, known differences in muscle composition, morphology, and function limit generalization of these findings to the muscles used during swallowing [31, 74].

Literature on fatigability during swallowing is sparse, and again, the evidence is mixed [75-80]. To date, studies examining swallowing-related fatigability have primarily focused on changes in tongue-to-palate strength and endurance of young and old healthy subjects, although fatigability of suprahyoid musculature involved in upper esophageal sphincter opening has also been studied during the head-lift exercise [81-83]. While this review focuses on swallowing, vocal fatigability is a topic that receives significant attention within the field of speech-language pathology e.g., [84-86] and is mentioned here given the overlapping musculature involved in swallowing and voicing. While an in-depth review of vocal fatigability is beyond the scope of this article, it is interesting to note that similar issues with standardization of definitions, terminology, and clinical identification exist within the field of voice. We direct readers to Welham \& Maclagan [87] and Hunter et al. [88] for in depth reviews of vocal fatigue.

For the purposes of this review, we focus the following discussion on studies that have examined lingual fatigability over the course of a meal, followed by studies examining lingual fatigability during intentionally fatiguing exercise tasks. 
Given that swallowing is a sub-maximal task, and the orofacial and pharyngeal muscles used during swallowing are predominated by slow-twitch, fatigue-resistant type I fiber types, it is reasonable to expect minimal fatigability of healthy swallowing musculature during a meal. Yet, in older adults, declines in anterior tongue strength over the course of a meal have been reported [76, 89]. Other studies, however, have found no effect of meal consumption on anterior tongue strength in older participants [75], and even increases in tongue strength post-meal [79]. Interestingly, the primary methodological difference between these studies was the type of meal consumed. Both Kays et al. [76] and Brates \& Molfenter [89] used a standardized meal consisting of half of a bagel with peanut butter, eight baby carrots, and a thin liquid beverage. The food and liquid textures in this standardized meal are likely more challenging as compared to the meals consumed in studies finding no declines in anterior tongue strength [75, 79], which used meals consistent with participants' normal diets (i.e. less challenging). Across these studies, the only one to find significant declines in posterior tongue strength or endurance after meal consumption was Kays et al. [76]. A conclusion that may be drawn from the available evidence is that elderly, non-dysphagic adults are not at risk for tongue fatigue when eating a meal consistent with their normal diet.

To understand the endurance capacity of the lingual musculature, Solomon et al. [77] had young healthy participants $(n=8)$ perform anterior tongue-to-palate presses until they could no longer sustain $50 \%$ of their baseline maximal tongue strength for three consecutive cycles. This fatigue threshold was reached after an average of 31.5 minutes of performing the task. The goal of this study was to explore fatigue-related changes in speech, and thus no swallowing parameters were included as outcome measures. However, the authors found significant differences in perceptual and acoustic speech parameters after the fatigue task.

Vanderwegen and Van Nuffelen [80] used a similar tongue-to-palate lingual fatigue protocol to compare differences in anterior and posterior tongue fatigability in healthy adults across three age groups (20-60 years old, $n=20$ and $70+$ years old, $n=20$ ). In contrast to Solomon et al., no participants ever fell below $50 \%$ of their baseline tongue pressures (exercise sessions were aborted after 30 minutes).

In one of the few studies on lingual fatigue in a disordered population, Solomon \& Robin [78] compared endurance and perceived effort during tongue-to-palate exercises between people with Parkinson's disease $(P D)(n=16)$ and healthy adults $(n=16)$. Perceived effort was significantly higher in the PD group, and the PD group reached task failure more quickly compared to the healthy group [78]. Taken together, the results of these studies confirm that the tongue is highly resistant to fatigue in healthy, non-dysphagic adults, and possibly less so in dysphagic populations.

Another muscle group that potentially plays a role in swallowing-related fatigue is the postural muscles of the trunk (e.g. external obliques, iliocostalis lumborum pars thoracis, internal oblique, lumbar multifidus, rectus abdominis, and thoracic erector spinae). In our clinician survey [46], some SLP respondents $(8 \%$; 8/96) reported postural changes as a clinical indicator of fatigue during swallowing assessments. Postural muscles are activated during upright seated position [90], which is the conventional eating position and is recommended for optimal safety and ease during mealtimes [91]. Fatigue of postural muscles has been demonstrated to negatively affect balance and functional tasks in older people [92]. Yet, the impact of fatigue of postural muscles on swallowing function is currently unknown and warrants further study. 


\subsection{Effects of Cognitive Fatigability on Swallowing}

A discussion about cognitive fatigability and swallowing/mealtime behavior with age is limited to the theoretical and anecdotal realms, given that, to our knowledge, this topic has not been empirically studied. However, the link between cognitive fatigue and swallowing warrants discussion due to the strong theoretical basis for their association.

In addition to requiring muscular endurance, the act of sitting down to independently consume a meal requires a minimum threshold of sustained attention and executive functioning if it is to be performed safely and continuously [93, 94]. Eating a meal is much more than simply chewing and swallowing. It represents an activity of daily living (ADL). ADLs are necessary, everyday behaviors and activities that require adequate executive functioning skills to maintain attention, selfmonitoring, self-regulation, and sequencing [95] in relation to homeostatic [96], environmental [97], and psychological factors [98]. Each of these factors represents a possible opportunity for disruption to safe and adequate meal consumption.

The importance of cognitive skills for facilitating safe and efficient swallowing is supported by evidence that dual-task paradigms (i.e. divided attention) can lead to changes in swallowing performance in healthy older individuals [99] and those with Parkinson's Disease [100, 101]. It is also reflected in the common clinical recommendation for patients to avoid distractions (e.g., environmental noise, conversation, interruptions) during mealtimes e.g., [102-104].

Executive control has been shown to decrease with aging, as evidenced by structural and functional neurochemical changes to the frontal lobe $[105,106]$, with attentional control being an early and highly affected function [107]. Brain regions that are involved in food processing have been found to be strongly modulated by attention focus, including the ventral striatum, premotor cortex, anterior cingulate cortex, insula/frontal operculum, ventrolateral prefrontal cortex and dorsolateral prefrontal cortex [108]. A comparison of swallowing-related brain activity on fMRI between young and old healthy adults has demonstrated increased activity across large regions of the superior and middle frontal lobes in older adults during swallowing, which are cortical areas associated with tasks requiring attention [109].

Cognitive fatigue refers to the effects of prolonged periods of cognitively demanding activity requiring sustained mental effort [110]. Cognitive fatigue can manifest as measurable increases in mental effort, reduced alertness, impaired learning and performance, and stress [111], and also as a lack of motivation and excitement to initiate or sustain task performance [112].

Cognitive fatigability in older adults has been demonstrated after prolonged attention on cognitively taxing tasks [113]. In all humans, cognitive resources are limited and must be allocated effectively and flexibly to adapt to varying cognitive demands. Thus, degraded performance occurs with increasing task demands [114]. While more "automatic" motor tasks such as walking or swallowing are considered to be immune to this type of dual-task deterioration in young and/or healthy individuals [115], increased cognitive demands have been shown to degrade motor performance in older adults [116] and those with neurological impairment/disease [117, 118], due to reduced cognitive resources to begin with, and requirement for more cognitive resources on tasks than would be used by young and/or neurologically healthy individuals. It is possible that the cognitive demands of everyday life may induce fatigue that affects mealtime behavior and/or swallowing performance in older adults and those with neurological disease, including individuals who suffer depression or other psychological disorders [119]. Mental weariness could result in a 
lack of motivation to complete a meal and could lead to declines in swallowing performance due to fatigue-induced changes in cognitive functions required for the deglutition process. Risks of cognitive fatigue may be magnified in dysphagic populations who have cognitive impairment (e.g. Alzheimer's Disease, dementia, Parkinson's Disease) and in those who practice recommended feeding strategies and postural maneuvers during meals that may further tax the cognitive system (e.g. performing a chin tuck with every swallow).

The potential relevance of cognitive fatigue further extends to the area of swallowing rehabilitation since therapeutic swallowing exercise programs are often rigorous and require multiple daily sets of varying swallowing exercises. Clinical recommendations often specify that patients should not perform swallowing exercises before meals. In addition to physical fatigue, it is possible that these exercises induce cognitive fatigue that further places patients at risk for impaired swallowing performance. Cognitive fatigue has been demonstrated to degrade neuromuscular performance in older adults [120,121], therefore effects of cognitive fatigability on motor fatigability of the swallowing mechanism may reduce exercise effectiveness in older patients.

\section{Perceived Fatigue During Swallowing: Subjective Changes in Performance}

Self-perceived fatigue refers to a person's subjective experience of fatigue, and is best described as a symptom [49, 53]. Fatigue is one of the most common symptoms reported by older adults [47], and self-report is one of the most common methods used by SLPs to identify swallowing-related fatigue, as we have found in a recent clinician survey [46].

There are many available scales and questionnaires that target generalized self-perceived fatigue (e.g. Fatigue Questionnaire [122], Fatigue Severity Scale [123], Multidimensional Assessment of Fatigue Scale [124]), though the operationalization of this symptom varies across scales [125]. Further, clinicians lack standardized methods for defining and identifying self-perceived fatigue [49]. The SWAL-QOL is a validated scale designed to assess dysphagia-related quality-of-life, and includes questions related to fatigue and sleep [126]. However, the creators of this scale note that the fatigue- and sleep-related items are not dysphagia-specific, and are rather designed to capture general quality-of-life factors. Therefore, when patients complain of swallowing-related fatigue, the specific and individualized characteristics of this symptom and how it impacts swallowing and eating experience remains unknown. For example, it is unclear how a sensation of tired masticatory muscles during chewing differentially impacts swallowing and eating compared to feelings of weariness or lack of motivation to eat, and which populations are at heightened risk for each. In order to better understand these factors, swallowing-related fatigue should be investigated with respect to the various dimensions that are traditionally used to characterize clinical symptoms such as pain (e.g. chronology, location, quality, quantity, setting, aggravating or alleviating factors, and associated manifestations) [127].

\section{Relationship between Performance fatigability and Perceived Fatigue During Swallowing}

Measurable fatigue during performance has not been found to be consistently correlated with the subjective experience of fatigue $[128,129]$. In fact, some studies show that young adults, despite having better resistance to fatigability, report higher levels of fatigue than older adults [130, 131]. This may be related to the way individuals self-regulate and control output to stay within a tolerable range of self-perceived fatigue [128]. For example, an individual with a low fatigability threshold 
(possibly due to functional impairment) may limit physical output and thus will not reach significant levels of perceived fatigue, whereas an individual with high functional capacity may not restrict physical output and will reach higher levels of perceived fatigue. In the context of swallowing and mealtime behaviors, this may be reflected in food texture and consistency choices, meal preparation time and effort, meal size, and/or meal duration.

A lack of correlation between perceived fatigue and fatigability is also demonstrated in patient populations including stroke [132], myasthenia gravis [133], muscular dystrophies [134], and peripheral neuropathy [134], with patients experiencing fatigue symptoms in the absence of significant measurable fatigue [132-134]. In patient populations, the relationship between these two constructs is complicated by mood and/or psychological conditions related to disease and disability [135], and patients may have difficulty distinguishing between muscle dysfunction due to neurological impairment (e.g. hemiparesis following stroke) versus fatigue symptoms [136]. The lack of correlation between fatigue and fatigability highlights the importance of normalizing measures of self-perceived fatigue to the activity context in which fatigue is experienced and to the specific population of interest.

The exercise physiology literature suggests that measures of self-perceived fatigue, or "sense-ofeffort" can be exploited for the purposes of maximizing muscle hypertrophy. Burd et al. [137] compared rates of protein synthesis when healthy subjects performed a leg extension exercise at varying loads (i.e. high load: $90 \%$ maximum capacity and low load: $30 \%$ maximum capacity) either for a fixed number of repetitions (normalized to load) or until volitional failure (i.e. fatigue threshold). They found that four hours post-exercise, myofibrillar protein synthesis showed a significant and similar response across the two loading conditions when performed to volitional failure, but at 24 hours post-exercise, the protein response was only sustained when the low load (30\% of maximum) was performed to volitional failure. A dose-dependent (repetitions $x$ load) effect was seen when repetition rate was fixed. These findings suggest that exercise performed to perceived fatigue is effective for inducing muscle hypertrophy, and lower loads (which can be performed for more repetitions before failure) may be superior to high loads for targeting increased force generation.

The neurophysiological basis for these findings is that, as a muscle fatigues, smaller motor units (innervating Type I slow-twitch, fatigue resistant fibers) cease firing, and larger motor units (innervating Type II fast-twitch, less fatigue-resistant fibers) have to be recruited if the person is to maintain force output [138]. It has been proposed that muscle adaptation is driven by maximum motor unit recruitment and contractile failure [139]. This may be optimally achieved using low-tomoderate loads at high repetitions, to allow time for failure of fatigue-resistant Type I fibers and subsequent recruitment of Type II fibers, which are more responsive to muscle hypertrophy [140].

The translation of this evidence to swallowing exercise protocols may be highly useful, given that for many exercises targeting the oropharyngeal musculature, an estimation of load is not practical or feasible in clinical settings, or in prescribed home-based regimens. This problem, and the associated dearth of evidence on optimal dosage for swallowing exercises, likely contributes to the high variability seen in swallowing exercise dosage recommendations [141]. Instead of prescribing a fixed dosage, having patients perform swallowing exercise repetitions until they reach their fatigue threshold (e.g. by using sense-of-effort scales) may improve standardization and tracking of exercise progress, enhance individually-tailored exercise programs, and better facilitate muscle 
hypertrophy to improve swallowing outcomes. However, much more evidence is needed to understand the role of self-perceived fatigue thresholds in swallowing rehabilitation and treatment.

\section{Clinical Implications and Future Directions}

Fatigue is a term that is commonly used to describe patient performance and/or subjective experience in the evaluation and management of dysphagia. Given the established association between fatigue and many dysphagia-causing diseases/disorders, SLPS and their professional associations such as ASHA are justifiably aware of and concerned about the potential impact of fatigue on swallowing performance and mealtime behavior. However, there is minimal agreement on and understanding of what constitutes swallowing-related fatigue, how it is identified and measured, who is at risk, and its impact on swallowing function, overall health, and quality of life.

In this review, we have proposed the application of a dichotomous framework [53] for conceptualizing swallowing related fatigue: performance fatigability and self-perceived fatigue symptoms. Given the broad scope of the term "fatigue" and the complexity of what it can describe, we believe that approaching future study and conceptualization of swallowing-related fatigue through this lens is optimal for clearly identifying gaps in current knowledge and designing experimental approaches for resolving them. Figure 2 illustrates a modification of this framework specifically for swallowing-related fatigue, which includes a subset of possible functional changes associated with swallowing-related fatigue that require further study (b). The clinical implications and future directions for each will be discussed in turn, beginning with performance fatigability of the muscles used in swallowing.

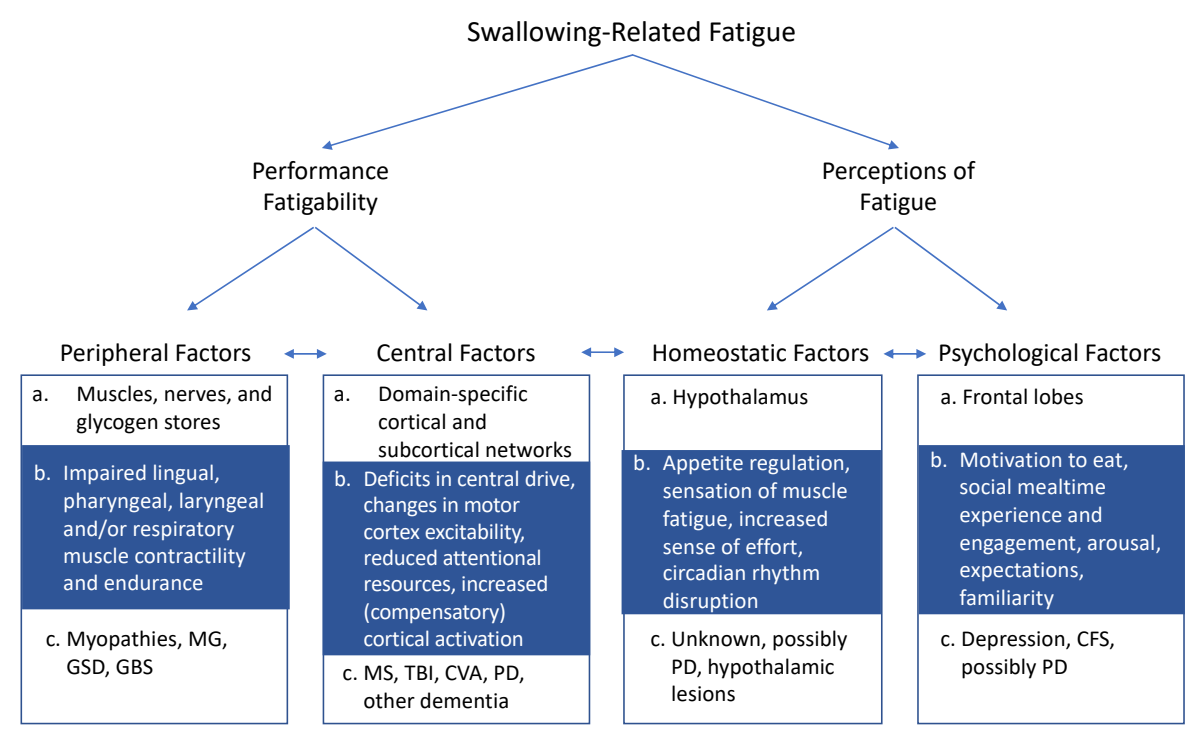

Figure 2 Figure adapted from Kluger BM, Krupp LB, Enoka RM. Fatigue and fatigability in neurologic illnesses: proposal for a unified taxonomy. Neurology. 2013; 80(4): 409-16, p. 412. Letters in boxes refer to the following: a) known neuroanatomic sites mediating this factor; b) possible factors associated with swallowing-related fatigue; c) pathologic states involving this factor. CFS = chronic fatigue syndrome; CVA = cerebrovascular accident; $E C$ = excitation/ contraction; GBS = Guillain-Barre Syndrome; GSD = glycogen storage diseases; $M G=$ myasthenia gravis; $M S=$ multiple sclerosis; $P D=$ Parkinson diseases; $\mathrm{TBI}$ = traumatic brain injury. 


\subsection{Performance Fatigability: Implications for Swallowing and Future Directions}

Though limited, the current evidence on swallowing muscle fatigability suggests that lingual endurance is very high in non-dysphagic individuals, though the anterior tongue may be more vulnerable to fatigue over the course of a meal in older adults. However, the scope of this conclusion is narrow, and, until further study, its clinical significance is unclear. Some evidence exists to suggest a limited association between anterior tongue strength and functional [142] or physiological swallowing outcomes [143]. Further, it is possible that other muscle groups involved in swallowing (i.e. respiratory, pharyngeal, and/or laryngeal muscles) fatigue before the tongue, resulting in very different consequences to swallowing performance. Imaging studies (e.g. using videofluoroscopy) are needed to investigate whether fatigue-induced changes are manifested as impaired swallowing function (such as aspiration and/or residue) or physiological changes in the pharynx (such as changes in measures of pharyngeal timing and/or extent of structural movement). Research on the effects of respiratory muscle fatigue (i.e. diaphragm, intercostal, and abdominal muscles) on respiratory-swallow patterning is also warranted. Fatigue could potentially disrupt the precise and crucial coordination of swallowing and breathing that is needed for airway protection during swallowing, particularly in populations that demonstrate aberrant respiratory-swallow coordination, such as Parkinson's Disease [144]. Logemann [145] recommended using a "stress test" involving videofluoroscopic observation of pre- and post-meal swallowing performance if fatigue is suspected. This method may be useful for identifying swallowing-related fatigue and its consequences, though there is a need for specification and standardization of criteria for "suspected fatigue", stress test protocols for optimally inducing fatigue, and quantifiable measures for confirming its occurrence.

As illustrated in Figure 2, performance fatigability can be caused by central or peripheral nervous system factors. It is important to distinguish between failures arising centrally versus peripherally as these have different etiologies and implications for rehabilitation. Yet, behavioral tasks such as stress-test protocols or maximal voluntary contractions (MVCs) during tongue-to-palate presses do not allow for distinction between these mechanisms. Solomon [78] has proposed that increased sense-of-effort ratings during tongue presses in adults with Parkinson's Disease may reflect central fatigue processes, given the central pathophysiology of this disease. Etiological information may serve as a helpful starting point for understanding factors involved in swallowing-related muscle fatigue, and muscle groups most at risk for fatigue during swallowing. Looking further, understanding how effects of cognitive fatigability, such as declines in sustained attention, relate to and interact with swallowing performance during a meal, may also help to tease apart central verses peripheral fatigue mechanisms. More research is needed to test these relationships.

The need for quantitative evidence of swallowing-related performance fatigability presents a scientific challenge due to the difficulty of directly measuring fatigue in the pharyngeal, laryngeal, and respiratory musculature. Traditional direct assessment of external skeletal muscle fatigue (e.g. by measuring force output on MVCs or using surface or intramuscular electrodes) is not feasible or ethical for internal application in live human subjects. Use of animal models is a compelling alternative, as is the application of indirect measures of fatigue (e.g. measurement of endurance time or electroencephalogram (EEG) readings). High resolution pharyngeal manometry $[146,147]$ could also be used to capture changes in pharyngeal pressures as a proxy for fatigue processes. Lastly, quantitative changes in swallowing biomechanics may be compared to measures of self- 
perceived fatigue, as has been done previously to understand lingual fatigue in non-dysphagic individuals and those with Parkinson's Disease [78].

Although surface electromyography (sEMG) has previously been used to measure swallowingrelated fatigue $[75,81,83]$, we caution against this application for two reasons. First, as mentioned, the external placement of sEMG electrodes may not fully capture muscle activity in all the submental musculature used during swallowing (including mylohyoid, geniohyoid, anterior belly of the digastric, and genioglossus), and activation patterns of these submental muscles may vary across individuals [148]. Second, a causal relationship between SEMG output and muscle fatigue is a subject of debate [73]. Studies that pool data across participants have shown that sEMG amplitudes gradually increase across repeated or sustained submaximal contractions [149, 150]. This may be explained by a rise in excitation rates and recruitment of a greater number of muscle fibers to maintain intensity as fatigue occurs [73]. Yet, within-subject data has shown that sEMG amplitudes in limb muscles may rise, fall, or stay the same as a muscle fatigues [151, 152]. This variable sEMG activity has also been observed in head and neck muscles (suprahyoid, infrahyoid, and sternocleidomastoid) during a head-lift exercise [83], with one of five subjects demonstrating large increases in mean frequency rates over the duration of the fatiguing task. This participant was excluded from analyses in this study by White et al. [83] due to these unexpected findings. Because sEMG amplitude represents neural activation (i.e. central fatigue processes), any mechanisms of fatigue occurring within the muscle itself (i.e. peripheral fatigue), which occur after the electrical activation that is registered on SEMG, will not be captured. Thus, between-person variability in mechanisms for fatigue, and variable changes in rate of force changes or muscle length during a given exercise task will result in variable fatigue-related SEMG readings. While this may prove useful for understanding central fatigue mechanisms only (and should be explored further), sEMG may not accurately reflect fatigue-induced changes in force output [73].

\subsection{Perceived Fatigue: Implications for Swallowing and Future Directions}

Our review of perceived fatigue has thus far focused on methods used to capture and assess fatigue symptomology, and its relationship to performance fatigability. In figure 2, we propose possible homeostatic and psychological factors that may contribute to the experience of swallowing and mealtime fatigue that represent areas for future study. The need to understand these factors highlights the complex and multidimensional nature of fatigue, which is represented in Figure 2 with bidirectional arrows between each factor within performance fatigability and perceived fatigue domains. Swallowing-related fatigue must be contextualized within the entire mealtime experience, given the interaction of homeostatic (e.g. hunger) and psychological factors (e.g. mood, motivation) to an experience of fatigue during swallowing. When a patient complains of fatigue during swallowing, there is more to consider than, for example, localized endurance of the lingual musculature. This complexity underscores the relevance of fatigue to the evaluation of dysphagia, which must consider patients holistically, beyond their ability to take a few sips of liquid without aspirating during a brief assessment.

There is a need to establish prevalence and characteristics of perceived swallowing-related fatigue with aging and across different dysphagic populations. Additionally, it is critical to understand the relationship between perceived swallowing-related fatigue and outcomes related to health, nutrition status, frailty status, and quality of life. This information will be useful for the 
development of much needed valid, swallowing-specific fatigue scales. Research into perceived fatigue should consider the proposed homeostatic and psychological factors proposed in Figure 2, such as how the mealtime social environment, arousal, mood, and appetite, contribute to feelings of fatigue reported by dysphagic patients. In addition to mealtime adequacy, safety, and health, these are issues that impact quality of life, which is central to the clinical rehabilitation and management of swallowing disorders.

Measures of perceived fatigue (e.g. sense-of-effort scales) should also be used to understand the relationship between perceived swallowing-related fatigue and performance fatigability under imaging, which may help to identify cut-off criteria in sense-of-effort for functional risk. The utility of sense-of-effort scales in swallowing exercise should also be explored to understand whether this can be used to standardize and optimize swallowing rehabilitation protocols.

\section{Conclusions}

SLPs who evaluate and treat swallowing disorders are trained to consider swallowing performance (i.e. adequate strength, skill, and coordination of the swallowing system to result in safe and efficient bolus passage), within the multidimensional, individualized, and nuanced context in which swallowing occurs. This includes a patient's overall health status and comorbidities, quality of life, personal preferences, cultural norms, and the mealtime context (and associated social context). Fatigue is only one aspect of this complex picture, but may play a role at every level of the swallowing and eating process. As we have discussed in this review, performance fatigability (in both motor and cognitive domains) and perceptions of fatigue are thus relevant to safe, effective, and optimal dysphagia management and rehabilitation, yet need to be further clarified through future study. More immediately and urgently, the establishment of a shared understanding and definition of swallowing-related fatigue, that is recognized and supported by official guidelines, is needed for dysphagia clinical care, both within the field of Speech Language Pathology and across disciplines working with this population. This shared framework will improve awareness and agreement on issues related to fatigue and its role in dysphagia evaluation and management, and will provide a much-needed foundation for future study in this area.

\section{Ethics Statement}

No human or animal subjects were involved in this study.

\section{Author Contributions}

DB: overall conception, primary role in drafting, and revised manuscript; SM: manuscript conception and revision; MT: manuscript conception and revision.

\section{Funding}

This work was partially funded by the ASHFoundation New Century Scholarship, awarded to the first author. 


\section{Competing Interests}

The authors have declared that no competing interests exist.

\section{References}

1. Gill TM, Desai MM, Gahbauer EA, Holford TR, Williams CS. Restricted activity among community-living older persons: Incidence, precipitants, and health care utilization. Ann Intern Med. 2001; 135: 313-321.

2. Okkes IM, Oskam SK, Lamberts $H$. The probability of specific diagnoses for patients presenting with common symptoms to Dutch family physicians. J Fam Pract. 2002; 51: 31-36.

3. Katz S. Assessing self-maintenance: Activities of daily living, mobility, and instrumental activities of daily living. J Am Geriatr Soc. 1983; 31: 721-727.

4. Baek Y, Jung K, Kim H, Lee S. Association between fatigue, pain, digestive problems, and sleep disturbances and individuals' health-related quality of life: A nationwide survey in South Korea. Health Qual Life Outcomes. 2020; 18: 159.

5. Avlund $\mathrm{K}$, Vass $\mathrm{M}$, Hendriksen $\mathrm{C}$. Onset of mobility disability among community-dwelling old men and women. The role of tiredness in daily activities. Age Ageing. 2003; 32: 579-584.

6. Avlund K, Damsgaard MT, Sakari-Rantala R, Laukkanen P, Schroll M. Tiredness in daily activities among nondisabled old people as determinant of onset of disability. J Clin Epidemiol. 2002; 55 : 965-973.

7. Irvine D, Vincent L, Graydon JE, Bubela N, Thompson L. The prevalence and correlates of fatigue in patients receiving treatment with chemotherapy and radiotherapy. A comparison with the fatigue experienced by healthy individuals. Cancer Nurs. 1994; 17: 367-378.

8. Glader EL, Stegmayr B, Asplund K. Poststroke fatigue: A 2-year follow-up study of stroke patients in Sweden. Stroke. 2002; 33: 1327-1333.

9. Penner IK, McDougall F, Brown TM, Slota C, Doward L, Julian L, et al. Exploring the impact of fatigue in progressive multiple sclerosis: A mixed-methods analysis. Mult Scler Relat Disord. 2020; 43: 102207.

10. Economou NT, Ilias I, Velentza L, Papachatzakis Y, Zarogoulidis P, Kallianos A, et al. Sleepiness, fatigue, anxiety and depression in chronic obstructive pulmonary disease and obstructive sleep apnea-overlap-syndrome, before and after continuous positive airways pressure therapy. PLoS ONE. 2018; 13: e0197342.

11. Wolfe F, Hawley DJ, Wilson K. The prevalence and meaning of fatigue in rheumatic disease. J Rheumatol. 1996; 23: 1407-1417.

12. Wijeratne $\mathrm{C}$, Hickie I, Brodaty $\mathrm{H}$. The characteristics of fatigue in an older primary care sample. J Psychosom Res. 2007; 62: 153-158.

13. Avlund K. Fatigue in older adults: An early indicator of the aging process? Aging Clin Exp Res. 2010; 22: 100-115.

14. Liao S, Ferrell BA. Fatigue in an older population. J Am Geriatr Soc. 2000; 48: 426-430.

15. Fried LP, Tangen CM, Walston J, Newman AB, Hirsch C, Gottdiener J, et al. Frailty in older adults: Evidence for a phenotype. J Gerontol A Biol Sci Med Sci. 2001; 56: M146-M157.

16. Kehler DS, Ferguson T, Stammers AN, Bohm C, Arora RC, Duhamel TA, et al. Prevalence of frailty in Canadians 18-79 years old in the Canadian health measures survey. BMC Geriatr. 2017; 17: 
28.

17. Gonzalez-Fernandez M, Humbert I, Winegrad H, Cappola AR, Fried LP. Dysphagia in old-old women: Prevalence as determined according to self-report and the 3-ounce water swallowing test. J Am Geriatr Soc. 2014; 62: 716-720.

18. van der Maarel-Wierink CD, Vanobbergen JN, Bronkhorst EM, Schols JM, de Baat C. Metaanalysis of dysphagia and aspiration pneumonia in frail elders. J Dent Res. 2011; 90: 1398-1404.

19. Bernstein M, Munoz N, Academy of N, Dietetics. Position of the academy of nutrition and dietetics: Food and nutrition for older adults: Promoting health and wellness. J Acad Nutr Diet. 2012; 112: 1255-1277.

20. Rozin P. Human food selection: Why do we know so little, and what can we do about it? Int J Obes. 1980; 4: 333-337.

21. Roberts SB. Energy regulation and aging: Recent findings and their implications. Nutr Rev. 2000; 58: 91-97.

22. Rolls BJ, McDermott TM. Effects of age on sensory-specific satiety. Am J Clin Nutr. 1991; 54: 988996.

23. Labeit B, Claus I, Muhle P, Regner L, Suntrup-Krueger S, Dziewas R, et al. Effect of cognitive and motor dual-task on oropharyngeal swallowing in Parkinson's disease. Eur J Neurol. 2021; 28: 754-762.

24. Burger S, Kayser-Jones JS, Bell JP. Malnutrition and dehydration in nursing homes: Key issues in prevention and treatment. Washington, DC: Commonwealth Fund; 2000.

25. Amarya $S$, Singh $K$, Sabharwal $M$. Changes during aging and their association with malnutrition. J Clin Gerontol Geriatr. 2015; 6: 78-84.

26. Acar Tek N, Karacil-Ermumcu MS. Determinants of health related quality of life in home dwelling elderly population: Appetite and nutritional status. J Nutr Health Aging. 2018; 22: 996-1002.

27. Perry L, McLaren S. An exploration of nutrition and eating disabilities in relation to quality of life at 6 months post-stroke. Health Soc Care Community. 2004; 12: 288-297.

28. LaCaille L. Eating Behavior. In Encyclopedia of Behavioral Medicine. New York, NY: Springer New York; 2013. pp.641-642.

29. Matsuo K, Palmer JB. Anatomy and physiology of feeding and swallowing: Normal and abnormal. Phys Med Rehabil Clin N Am. 2008; 19: 691-707.

30. American College of Sports Medicine. Guidelines for exercise testing and prescription. 4th ed. Philadelphia: Lea \& Febiger; 1991.

31. Kent RD. The uniqueness of speech among motor systems. Clin Linguist Phon. 2004; 18: 495505.

32. Hoh JF. Laryngeal muscle fibre types. Acta Physiol Scand. 2005; 183: 133-149.

33. Mu L, Sanders I. Neuromuscular compartments and fiber-type regionalization in the human inferior pharyngeal constrictor muscle. Anat Rec. 2001; 264: 367-377.

34. Polla B, D'Antona G, Bottinelli R, Reggiani C. Respiratory muscle fibres: Specialisation and plasticity. Thorax. 2004; 59: 808-817.

35. Stal P, Marklund S, Thornell LE, De Paul R, Eriksson PO. Fibre composition of human intrinsic tongue muscles. Cells Tissues Organs. 2003; 173: 147-161.

36. Donini LM, Savina C, Cannella C. Eating habits and appetite control in the elderly: The anorexia of aging. Int Psychogeriatr. 2003; 15: 73-87.

37. Engel JH, Siewerdt F, Jackson R, Akobundu U, Wait C, Sahyoun N. Hardiness, depression, and 
emotional well-being and their association with appetite in older adults. J Am Geriatr Soc. 2011; 59: 482-487.

38. Leder SB, Suiter DM, Warner HL, Acton LM, Siegel MD. Safe initiation of oral diets in hospitalized patients based on passing a 3-ounce $(90 \mathrm{cc}$ ) water swallow challenge protocol. QJM. 2012; 105 : 257-263.

39. Burkhead LM, Sapienza CM, Rosenbek JC. Strength-training exercise in dysphagia rehabilitation: Principles, procedures, and directions for future research. Dysphagia. 2007; 22: 251-265.

40. Robbins J, Kays SA, Gangnon RE, Hind JA, Hewitt AL, Gentry LR, et al. The effects of lingual exercise in stroke patients with dysphagia. Arch Phys Med Rehabil. 2007; 88: 150-158.

41. Yeates EM, Molfenter SM, Steele CM. Improvements in tongue strength and pressuregeneration precision following a tongue-pressure training protocol in older individuals with dysphagia: Three case reports. Clin Interv Aging. 2008; 3: 735-747.

42. Hila A, Castell JA, Castell DO. Pharyngeal and upper esophageal sphincter manometry in the evaluation of dysphagia. J Clin Gastroenterol. 2001; 33: 355-361.

43. Leonard R, Belafsky PC, Rees CJ. Relationship between fluoroscopic and manometric measures of pharyngeal constriction: The pharyngeal constriction ratio. Ann Otol Rhinol Laryngol. 2006; 115: 897-901.

44. Athukorala RP, Jones RD, Sella O, Huckabee ML. Skill training for swallowing rehabilitation in patients with Parkinson's disease. Arch Phys Med Rehabil. 2014; 95: 1374-1382.

45. Huckabee ML, Macrae P. Rethinking rehab: Skill-based training for swallowing impairment. Dysphagia. 2014; 23: 46-53.

46. Brates D, Namasivayam-MacDonald AM, Molfenter SM. Survey of clinician perspectives and practices regarding swallowing-related fatigue. Am J Speech Lang Pathol. in press.

47. Egerton T. Self-reported aging-related fatigue: A concept description and its relevance to physical therapist practice. Phys Ther. 2013; 93: 1403-1413.

48. Murphy S, Niemiec SS. Aging, Fatigue, and fatigability: Implications for occupational and physical therapists. Curr Geriatr Rep. 2014; 3: 135-141.

49. Hardy SE, Studenski SA. Qualities of fatigue and associated chronic conditions among older adults. J Pain Symptom Manage. 2010; 39: 1033-1042.

50. Solomon NP. What is orofacial fatigue and how does it affect function for swallowing and speech? Semin Speech Lang. 2006; 27: 268-282.

51. Solomon NP. Assessment of tongue weakness and fatigue. Int J Orofacial Myology. 2004; 30: 819.

52. American Speech-Language-Hearing Association. Practice Dysphagia [Internet]. Rockville, MA: American Speech-Language-Hearing Association; [cited date 2019 September 26th]. Available from: https://www.asha.org/Practice-Portal/Clinical-Topics/Pediatric-Dysphagia/.

53. Kluger BM, Krupp LB, Enoka RM. Fatigue and fatigability in neurologic illnesses: Proposal for a unified taxonomy. Neurology. 2013; 80: 409-416.

54. Goodpaster BH, Park SW, Harris TB, Kritchevsky SB, Nevitt M, Schwartz AV, et al. The loss of skeletal muscle strength, mass, and quality in older adults: The health, aging and body composition study. J Gerontol A Biol Sci Med Sci. 2006; 61: 1059-1064.

55. Harris T. Muscle mass and strength: Relation to function in population studies. J Nutr. 1997; 127: 1004S-1006S.

56. Metter EJ, Conwit R, Tobin J, Fozard JL. Age-associated loss of power and strength in the upper 
extremities in women and men. J Gerontol A Biol Sci Med Sci. 1997; 52: B267-B276.

57. Hara K, Tohara H, Kobayashi K, Yamaguchi K, Yoshimi K, Nakane A, et al. Age-related declines in the swallowing muscle strength of men and women aged 20-89 years: A cross-sectional study on tongue pressure and jaw-opening force in 980 subjects. Arch Gerontol Geriatr. 2018; 78: 6470.

58. Crow HC, Ship JA. Tongue strength and endurance in different aged individuals. J Gerontol A Biol Sci Med Sci. 1996; 51: M247-M250.

59. Ratkevicius A, Skurvydas A, Lexell J. Submaximal-exercise-induced impairment of human muscle to develop and maintain force at low frequencies of electrical stimulation. Eur J Appl Physiol Occup Physiol. 1995; 70: 294-300.

60. Bemben MG, Massey BH, Bemben DA, Misner JE, Boileau RA. Isometric intermittent endurance of four muscle groups in men aged 20-74 yr. Med Sci Sports Exerc. 1996; 28: 145-154.

61. Ditor DS, Hicks AL. The effect of age and gender on the relative fatigability of the human adductor pollicis muscle. Can J Physiol Pharmacol. 2000; 78: 781-790.

62. Lanza IR, Russ DW, Kent-Braun JA. Age-related enhancement of fatigue resistance is evident in men during both isometric and dynamic tasks. J Appl Physiol. 2004; 97: 967-975.

63. Aniansson A, Grimby G, Hedberg M, Rungren A, Sperling L. Muscle function in old age. Scand J Rehabil Med Suppl. 1978; 6: 43-49.

64. Laforest S, St-Pierre DM, Cyr J, Gayton D. Effects of age and regular exercise on muscle strength and endurance. Eur J Appl Physiol Occup Physiol. 1990; 60: 104-111.

65. Lindstrom B, Lexell J, Gerdle B, Downham D. Skeletal muscle fatigue and endurance in young and old men and women. J Gerontol A Biol Sci Med Sci. 1997; 52: B59-B66.

66. Baudry S, Klass M, Pasquet B, Duchateau J. Age-related fatigability of the ankle dorsiflexor muscles during concentric and eccentric contractions. Eur J Appl Physiol. 2007; 100: 515-525.

67. Cupido CM, Hicks AL, Martin J. Neuromuscular fatigue during repetitive stimulation in elderly and young adults. Eur J Appl Physiol Occup Physiol. 1992; 65: 567-572.

68. Davies CT, White MJ. Contractile properties of elderly human triceps surae. Gerontology. 1983; 29: 19-25.

69. McNeil CJ, Rice CL. Fatigability is increased with age during velocity-dependent contractions of the dorsiflexors. J Gerontol A Biol Sci Med Sci. 2007; 62: 624-629.

70. Rawson ES. Enhanced fatigue resistance in older adults during repeated sets of intermittent contractions. J Strength Cond Res. 2010; 24: 251-256.

71. Enoka RM, Duchateau J. Muscle fatigue: What, why and how it influences muscle function. J Physiol. 2008; 586: 11-23.

72. Gandevia SC. Spinal and supraspinal factors in human muscle fatigue. Physiol Rev. 2001; 81: 1725-1789.

73. Vollestad NK. Measurement of human muscle fatigue. J Neurosci Methods. 1997; 74: 219-227.

74. Connor NP, Ota F, Nagai H, Russell JA, Leverson G. Differences in age-related alterations in muscle contraction properties in rat tongue and hindlimb. J Speech Lang Hear Res. 2008; 51: 818-827.

75. Hiramatsu T, Kataoka $\mathrm{H}$, Osaki M, Hagino $\mathrm{H}$. Effect of aging on oral and swallowing function after meal consumption. Clin Interv Aging. 2015; 10: 229-235.

76. Kays SA, Hind JA, Gangnon RE, Robbins J. Effects of dining on tongue endurance and swallowingrelated outcomes. J Speech Lang Hear Res. 2010; 53: 898-907. 
77. Solomon NP. Changes in normal speech after fatiguing the tongue. J Speech Lang Hear Res. 2000; 43: 1416-1428.

78. Solomon NP, Robin DA. Perceptions of effort during handgrip and tongue elevation in Parkinson's disease. Parkinsonism Relat Disord. 2005; 11: 353-361.

79. Heidi A. Van Ravenhorst-Bell. Changes in tongue strength and endurance after a typical meal in healthy older adults in a continuing care community. Proceedings of the 8th Annual Symposium: Graduate Research and Scholarly Projects; 2012 April 18th; Wichita, KS, USA. Wichita, KS: Wichita State University. pp.141-142.

80. Vanderwegen J, Van Nuffelen G. Evaluation of different fatigue-inducing paradigms (FP) on maximum isometric pressures (MIP) of the anterior tongue in healthy adults and elderly. Dublin, Ireland: Paper presented at the European Society for Swallowing Disorders; 2018.

81. Ferdjallah M, Wertsch JJ, Shaker R. Spectral analysis of surface electromyography (EMG) of upper esophageal sphincter-opening muscles during head lift exercise. J Rehabil Res Dev. 2000; 37: $335-340$.

82. Jurell KC, Shaker R, Mazur A, Haig AJ, Wertsch JJ. Spectral analysis to evaluate hyoid muscles involvement in neck exercise. Muscle Nerve. 1996; 19: 1224.

83. White KT, Easterling C, Roberts N, Wertsch J, Shaker R. Fatigue analysis before and after shaker exercise: Physiologic tool for exercise design. Dysphagia. 2008; 23: 385-391.

84. Gotaas C, Starr CD. Vocal fatigue among teachers. Folia Phoniatr Logop. 1993; 45: 120-129.

85. Sander EK, Ripich DE. Vocal fatigue. Ann Otol Rhinol Laryngol. 1983; 92: 141-145.

86. Solomon NP. Vocal fatigue and its relation to vocal hyperfunction dagger. Int J Speech Lang Pathol. 2008; 10: 254-266.

87. Welham NV, Maclagan MA. Vocal fatigue: Current knowledge and future directions. J Voice. 2003; 17: 21-30.

88. Hunter EJ, Cantor-Cutiva LC, van Leer E, van Mersbergen M, Nanjundeswaran CD, Bottalico P, et al. Toward a consensus description of vocal effort, vocal load, vocal loading, and vocal fatigue. J Speech Lang Hear Res. 2020; 63: 509-532.

89. Brates D, Molfenter S. The Influence of Age, Eating a Meal, and Systematic Fatigue on Swallowing and Mealtime Parameters. Dysphagia. 2021. Doi: 10.1007/s00455-020-10242-8.

90. O'Sullivan PB, Dankaerts W, Burnett AF, Farrell GT, Jefford E, Naylor CS, et al. Effect of different upright sitting postures on spinal-pelvic curvature and trunk muscle activation in a pain-free population. Spine. 2006; 31: E707-E712.

91. Alghadir AH, Zafar H, Al-Eisa ES, Iqbal ZA. Effect of posture on swallowing. Afr Health Sci. 2017; 17: 133-137.

92. Helbostad JL, Sturnieks DL, Menant J, Delbaere K, Lord SR, Pijnappels M. Consequences of lower extremity and trunk muscle fatigue on balance and functional tasks in older people: A systematic literature review. BMC Geriatr. 2010; 10: 56.

93. Chang CC, Roberts BL. Feeding difficulty in older adults with dementia. J Clin Nurs. 2008; 17: 2266-2274.

94. Lee KM, Song JA. Factors influencing the degree of eating ability among people with dementia. J Clin Nurs. 2015; 24: 1707-1717.

95. Cahn-Weiner DA, Malloy PF, Boyle PA, Marran M, Salloway S. Prediction of functional status from neuropsychological tests in community-dwelling elderly individuals. Clin Neuropsychol. 2000; 14: 187-195. 
96. Gibson AS, Swart J, Tucker R. The interaction of psychological and physiological homeostatic drives and role of general control principles in the regulation of physiological systems, exercise and the fatigue process-the integrative governor theory. Eur J Sport Sci. 2018; 18: 25-36.

97. Bruderer-Hofstetter M, Sikkes SAM, Munzer T, Niedermann K. Development of a model on factors affecting instrumental activities of daily living in people with mild cognitive impairmenta Delphi study. BMC Neurol. 2020; 20: 264.

98. Bergua V, Bouisson J, Dartigues JF, Swendsen J, Fabrigoule C, Peres K, et al. Restriction in instrumental activities of daily living in older persons: Association with preferences for routines and psychological vulnerability. Int J Aging Hum Dev. 2013; 77: 309-329.

99. Brodsky MB, McNeil MR, Martin-Harris B, Palmer CV, Grayhack JP, Abbott KV. Effects of divided attention on swallowing in healthy participants. Dysphagia. 2012; 27: 307-317.

100.Troche MS, Okun MS, Rosenbek JC, Altmann LJ, Sapienza CM. Attentional resource allocation and swallowing safety in Parkinson's disease: A dual task study. Parkinsonism Relat Disord. 2014; 20: 439-443.

101.Brodsky MB, Verdolini Abbott K, McNeil MR, Palmer CV, Grayhack JP, Martin-Harris B. Effects of divided attention on swallowing in persons with idiopathic Parkinson's disease. Dysphagia. 2012; 27: 390-400.

102.Aselage MB, Amella EJ, Watson R. State of the science: Alleviating mealtime difficulties in nursing home residents with dementia. Nurs Outlook. 2011; 59: 210-214.

103.Morris H. Adapting mealtimes to residents with dysphagia. Nursing and Residential Care. 2018; 20: 610-614.

104.Samuels R, Chadwick DD. Predictors of asphyxiation risk in adults with intellectual disabilities and dysphagia. J Intellect Disabil Res. 2006; 50: 362-370.

105.Driscoll I, Davatzikos C, An Y, Wu X, Shen D, Kraut M, et al. Longitudinal pattern of regional brain volume change differentiates normal aging from MCl. Neurology. 2009; 72: 1906-1913.

106.Peters A, Sethares C, Luebke Jl. Synapses are lost during aging in the primate prefrontal cortex. Neuroscience. 2008; 152: 970-981.

107.Jurado $M B$, Rosselli $M$. The elusive nature of executive functions: A review of our current understanding. Neuropsychol Rev. 2007; 17: 213-233.

108.Siep N, Roefs A, Roebroeck A, Havermans R, Bonte ML, Jansen A. Hunger is the best spice: An $f M R I$ study of the effects of attention, hunger and calorie content on food reward processing in the amygdala and orbitofrontal cortex. Behav Brain Res. 2009; 198: 149-158.

109. Humbert IA, Fitzgerald ME, McLaren DG, Johnson S, Porcaro E, Kosmatka K, et al. Neurophysiology of swallowing: Effects of age and bolus type. Neurolmage. 2009; 44: 982-991.

110.Lorist MM, Boksem MA, Ridderinkhof KR. Impaired cognitive control and reduced cingulate activity during mental fatigue. Brain Res Cogn Brain Res. 2005; 24: 199-205.

111.Ackerman PL. Cognitive fatigue: Multidisciplinary perspectives on current research and future applications. Washington, DC: American Psychological Association; 2011. pp.11-43.

112.Grandjean E. Fatigue in industry. Br J Ind Med. 1979; 36: 175-186.

113.Arnau S, Mockel T, Rinkenauer G, Wascher $E$. The interconnection of mental fatigue and aging: An EEG study. Int J Psychophysiol. 2017; 117: 17-25.

114.Franconeri SL, Alvarez GA, Cavanagh P. Flexible cognitive resources: Competitive content maps for attention and memory. Trends Cogn Sci. 2013; 17: 134-141.

115. Haggard P, Cockburn J, Cock J, Fordham C, Wade D. Interference between gait and cognitive 
tasks in a rehabilitating neurological population. J Neurol Neurosurg Psychiatry. 2000; 69: 479486.

116. Maylor EA, Wing AM. Age differences in postural stability are increased by additional cognitive demands. J Gerontol B Psychol Sci Soc Sci. 1996; 51: P143-P154.

117. Camicioli R, Howieson D, Lehman S, Kaye J. Talking while walking: The effect of a dual task in aging and Alzheimer's disease. Neurology. 1997; 48: 955-958.

118. Marshall SC, Grinnell D, Heisel B, Newall A, Hunt L. Attentional deficits in stroke patients: A visual dual task experiment. Arch Phys Med Rehabil. 1997; 78: 7-12.

119.Donini LM, Poggiogalle E, Piredda M, Pinto A, Barbagallo M, Cucinotta D, et al. Anorexia and eating patterns in the elderly. PLoS ONE. 2013; 8: e63539.

120.Clark BC, Taylor JL. Age-related changes in motor cortical properties and voluntary activation of skeletal muscle. Curr Aging Sci. 2011; 4: 192-199.

121.Shortz AE, Mehta RK. Cognitive challenges, aging, and neuromuscular fatigue. Physiol Behav. 2017; 170: 19-26.

122.Chalder T, Berelowitz G, Pawlikowska T, Watts L, Wessely S, Wright D, et al. Development of a fatigue scale. J Psychosom Res. 1993; 37: 147-153.

123.Krupp LB, LaRocca NG, Muir-Nash J, Steinberg AD. The fatigue severity scale. Application to patients with multiple sclerosis and systemic lupus erythematosus. Arch Neurol. 1989; 46: 11211123.

124.Piper BF, Lindsey AM, Dodd MJ, Ferketich S, Paul SM, Weller S. The development of an instrument to measure the subjective dimension of fatigue. In Management of Pain, Fatigue and Nausea. London: Macmillan Education UK; 1989. pp.199-208.

125.Knoop V, Costenoble A, Vella Azzopardi R, Vermeiren S, Debain A, Jansen B, et al. The operationalization of fatigue in frailty scales: A systematic review. Ageing Res Rev. 2019; 53: 100911.

126. McHorney CA, Robbins J, Lomax K, Rosenbek JC, Chignell K, Kramer AE, et al. The SWAL-QOL and SWAL-CARE outcomes tool for oropharyngeal dysphagia in adults: III. Documentation of reliability and validity. Dysphagia. 2002; 17: 97-114.

127.Engel GL. What if music students were taught to play their instruments as medical students are taught to interview? Pharos Alpha Omega Alpha Honor Med Soc. 1982; 45: 12-13.

128.Eldadah BA. Fatigue and fatigability in older adults. PM R. 2010; 2: 406-413.

129.Lou JS, Kearns G, Benice T, Oken B, Sexton G, Nutt J. Levodopa improves physical fatigue in Parkinson's disease: A double-blind, placebo-controlled, crossover study. Mov Disord. 2003; 18: 1108-1114.

130.Aggarwal VR, McBeth J, Zakrzewska JM, Lunt M, Macfarlane GJ. The epidemiology of chronic syndromes that are frequently unexplained: do they have common associated factors? Int J Epidemiol. 2006; 35: 468-476.

131.Fuhrer R, Wessely S. The epidemiology of fatigue and depression: A French primary-care study. Psychol Med. 1995; 25: 895-905.

132. Rahamatali M, De Bont N, Valet M, Halkin V, Hanson P, Deltombe T, et al. Post-stroke fatigue: How it relates to motor fatigability and other modifiable factors in people with chronic stroke. Acta Neurol Belg. 2021; 121: 181-189.

133.Jordan B, Mehl T, Schweden TLK, Menge U, Zierz S. Assessment of physical fatigability and fatigue perception in myasthenia gravis. Muscle Nerve. 2017; 55: 657-663. 
134.Schillings ML, Kalkman JS, Janssen HM, van Engelen BG, Bleijenberg G, Zwarts MJ. Experienced and physiological fatigue in neuromuscular disorders. Clin Neurophysiol. 2007; 118: 292-300.

135.Dobkin BH. Fatigue versus activity-dependent fatigability in patients with central or peripheral motor impairments. Neurorehabil Neural Repair. 2008; 22: 105-110.

136.Mead G, Lynch J, Greig C, Young A, Lewis S, Sharpe M. Evaluation of fatigue scales in stroke patients. Stroke. 2007; 38: 2090-2095.

137.Burd NA, West DW, Staples AW, Atherton PJ, Baker JM, Moore DR, et al. Low-load high volume resistance exercise stimulates muscle protein synthesis more than high-load low volume resistance exercise in young men. PLoS ONE. 2010; 5: e12033.

138.Dorfman LJ, Howard JE, McGill KC. Triphasic behavioral response of motor units to submaximal fatiguing exercise. Muscle Nerve. 1990; 13: 621-628.

139. Morton RW, McGlory C, Phillips SM. Nutritional interventions to augment resistance traininginduced skeletal muscle hypertrophy. Front Physiol. 2015; 6: 245.

140.Powers SK, Howley ET. Exercise physiology: Theory and application to fitness and performance. 8th ed. New York, NY: McGraw Hill; 2012.

141. Krekeler BN, Rowe LM, Connor NP. Dose in exercise-based dysphagia therapies: A scoping review. Dysphagia. 2020.

142. Molfenter SM, Brates D, Herzberg E, Noorani M, Lazarus C. The swallowing profile of healthy aging adults: Comparing noninvasive swallow tests to videofluoroscopic measures of safety and efficiency. J Speech Lang Hear Res. 2018; 61: 1603-1612.

143. Molfenter SM, Lenell C, Lazarus CL. Volumetric changes to the pharynx in healthy aging: Consequence for pharyngeal swallow mechanics and function. Dysphagia. 2019; 34: 129-137.

144.Troche MS, Huebner I, Rosenbek JC, Okun MS, Sapienza CM. Respiratory-swallowing coordination and swallowing safety in patients with Parkinson's disease. Dysphagia. 2011; 26: 218-224.

145.Logemann JA. Evaluation and treatment of swallowing disorders. 2nd ed. Austin, TX: Pro-Ed; 1998.

146. Knigge MA, Thibeault S, McCulloch TM. Implementation of high-resolution manometry in the clinical practice of speech language pathology. Dysphagia. 2014; 29: 2-16.

147.Omari TI, Ciucci M, Gozdzikowska K, Hernandez E, Hutcheson K, Jones C, et al. High-resolution pharyngeal manometry and impedance: Protocols and metrics-recommendations of a highresolution pharyngeal manometry international working group. Dysphagia. 2020; 35: 281-295.

148.Palmer PM, Luschei ES, Jaffe D, McCulloch TM. Contributions of individual muscles to the submental surface electromyogram during swallowing. J Speech Lang Hear Res. 1999; 42: 13781391.

149. Fallentin N, Jorgensen $\mathrm{K}$, Simonsen EB. Motor unit recruitment during prolonged isometric contractions. Eur J Appl Physiol Occup Physiol. 1993; 67: 335-341.

150. Maton B, Gamet D. The fatigability of two agonistic muscles in human isometric voluntary submaximal contraction: an EMG study. II. Motor unit firing rate and recruitment. Eur J Appl Physiol Occup Physiol. 1989; 58: 369-374.

151. Mengshoel AM, Saugen E, Forre O, Vollestad NK. Muscle fatigue in early fibromyalgia. J Rheumatol. 1995; 22: 143-150.

152.Zijdewind I, Kernell D, Kukulka CG. Spatial differences in fatigue-associated electromyographic behaviour of the human first dorsal interosseus muscle. J Physiol. 1995; 483: 499-509. 


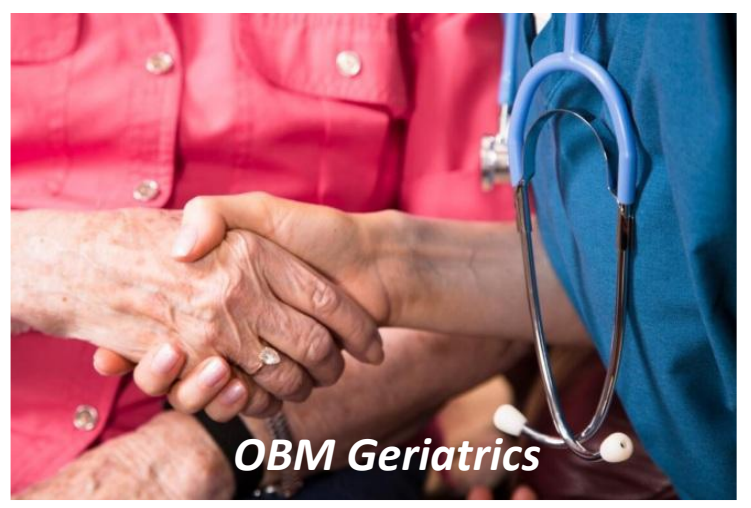

Enjoy OBM Geriatrics by:

1. Submitting a manuscript

2. Joining in volunteer reviewer bank

3. Joining Editorial Board

4. Guest editing a special issue

For more details, please visit:

http://www.lidsen.com/journals/geriatrics 